\title{
Titanium plasma source for capillary discharge extreme ultraviolet lasers
}

\author{
M. Shuker*, A. Ben-kish, A. Fisher, and A. Ron \\ Department of Physics, Technion - Israel Inst. of Tech., Haifa 32000, Israel
}

\begin{abstract}
A technique to generate jets of pure Titanium plasma is presented. A Ti wire is exploded in an Alumina capillary sealed with 1atm. of air inside. The generated plasma emerges from the capillary (to a high-vacuum environment) by ripping a thin Ti foil that seals one of the capillary ends. The generated plasma jets have a velocity of up to $4.5 \pm 0.5 \mathrm{~mm} / \mu \mathrm{s}$, an electron temperature of $1.5 \pm 0.5 \mathrm{eV}$ and a ion density of $2.7 \pm 1 \times 10^{17} / \mathrm{cc}$. The plasma source was designed for a capillary discharge extreme ultraviolet laser experiment, but might also be useful to other application such as a target for Z-pinch experiments.

PACS numbers: 52.25.-b,52.3.-q,52.50.-b
\end{abstract}

Among several techniques explored for achieving short wavelength lasing $\frac{1}{2}$, the capillary discharge has the advantage of being relatively simple, efficient and compact ${ }^{2}, \frac{3}{3}$. This technique is based on an electrical discharge preformed on low density gaseous targets in a dielectric capillary. Recently, a capillary discharge extreme ultraviolet (XUV) laser was realized in the $3 P \rightarrow 3 S$ transition of Ne-like Ar ions at $46.9 \mathrm{~nm}$. This laser was initially demonstrated by Rocca and his colleagues ${ }^{2}$, and several years later by other groups $\frac{4}{,}, \frac{6}{2}$. One of the challenges in scaling the capillary discharge laser to shorter wavelength is the formation of the initial plasma target. The target should be a capillary uniformly filled with low density $\left(N_{i} \cong 10^{16} \mathrm{par} / \mathrm{cc}\right)$, pre-ionized gas or vapor. This can be easily achieved with gases (or liquids with high vapor pressure ${ }^{7}$ ), however targets from materials that are solid at room temperature are more difficult to implement. Furthermore, high purity and uniformity targets are required as the laser is sensitive to instabilities.

Several possible techniques to create suitable plasma targets were previously explored. Ablative capillaries were used as the main capillary ${ }^{8}$ or as a secondary capillary shooting a jet of plasma into the main capillary ${ }^{9}$. The ablation can take place during the main current pulse or by adding a pre-pulse. In the case the required material is a conductor, an insulating compound must be used and an impure plasma is created (for example, a pressed $\mathrm{TiH}_{2}$ capillary was used to create a Ti plasma target ${ }^{8}$ ). Another possible solution is generating the plasma by ablating the capillary electrodes 10,11 . This technique is suitable for conductors, but is not efficient and might result in ablating part of the capillary walls as well.

In this letter we address the problem of creating a metallic plasma source. For our experiments we used Ti whose Ne-like ion lase at $32.6 \mathrm{~nm}$. We adopt the concept of the secondary capillary ${ }^{9}$, and suggest using an exploding wire in the secondary capillary to produce the plasma (instead of ablating the walls or the electrodes). The exploding wire has the advantages of being very efficient and producing high purity Ti plasma (its major draw-

*Email address: shuker@physics.technion.ac.il back is being a single-shot device). We note that the plasma jet must be created in a vacuum environment (atmospheric density is a few order of magnitude higher than the required density of the active atoms).

In order to determine the desired properties of the $\mathrm{Ti}$ plasma target (i.e. the properties just prior to the onset of the main current pulse), the dynamical process of the capillary discharge must be considered. We used a one dimensional magneto-hydrodynamical (MHD) $\operatorname{code}^{12}$, to model the capillary discharge experiment and to infer the required properties of the $T i$ plasma target. In all the calculations below we used the parameters of our pulsed power system ${ }^{4}$, that can produce peak currents of $40-130 K A$ with half-cycle time of $100-120 \mathrm{~ns}$. In a series of calculations we found suitable parameters of the plasma target that will result in a Ne-like Ti XUV laser. The required plasma target is a capillary filled with pre-ionized $\left(T_{e}=T_{i}=0.1-0.5 \mathrm{eV}\right)$ Ti plasma, with ion density of $N_{i}=1-4 \times 10^{16} / c c$. Therefore, the plasma jet from the secondary capillary must have a substantially higher density $\left(>10^{17} / c c\right)$ to create a suitable plasma target (it is very simple to reduce the density in the main capillary by a suitable flow geometry $\left.{ }^{13}\right)$.

The experimental system for generating the Ti plasma target consisted of a low energy discharge system with a $10 \mu F$ capacitor that was typically charged to $2 k V$. A low inductance semi-rigid coax cable was used to transfer the electrical energy to the secondary capillary. The total inductance of the electrical system was $900 \mathrm{nH}$ and the typical half-cycle duration was $10 \mu \mathrm{s}$. The peak currents in the system were in the range $0.5-2 k A$ (depending on the charging voltage and the capillary setup). The capillary consisted of a plastic structure with an alumina insert. The inner diameter of the capillary was $1 \mathrm{~mm}$, and its length was $10-30 \mathrm{~mm}$. The electrodes of the capillary were made of $\mathrm{Ti}$ to minimize the contamination of the Ti plasma. One of the electrodes had a concentric hole with $1 \mathrm{~mm}$ diameter (through which the plasma jet will emerge). The wire in the capillary was a high purity $\mathrm{Ti}$ wire of $125 \mu \mathrm{m}$ diameter. The experiments were constructed inside a vacuum chamber - and preformed in various environments ranging from atmospheric pressure to $10^{-6}$ Torr. Electrical measurements during the wire 


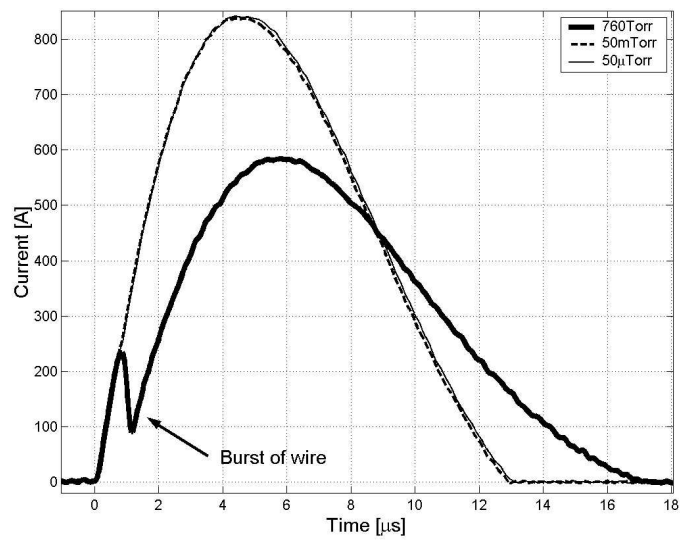

FIG. 1: Current signals of exploding wire in atmospheric pressure (thick line), in 50mTorr (dashed line) and in 50mTorr (thin line). A signature of the wire explosion is evident only in atmospheric pressure.

explosion were performed with a commercial Rogovsky coil and a Tektronix 1:1000 high voltage probe. A fast visible-light camera was used to study the flow of the plasma jet from the secondary capillary and its propagation towards the main capillary. The plasma properties (composition, electronic density and temperature) were inferred from visible light spectroscopy measurements using a spectrometer coupled to a gated CCD camera.

The exploding wire technique is a well known method to generate high-temperature plasmas. However, there is a major difficulty in realizing this technique in vacuum environment (as required in our case). Figure 1 depicts the current signals measured in three experiments performed in our system at different ambient pressure.

In atmospheric pressure a clear signature of the wire explosion is evident (the current sharply drops and increases again shortly afterwards). However, at ambient pressures $50 \mathrm{mT}$ Torr, $50 \mu$ Torr the current shows no evidence of the wire explosion. Fast photography experiments confirmed that the wire did not explode (in fact it stayed connected to the electrodes and intact during the entire electrical discharge), and consequently no plasma was formed. The reason for this phenomena in vacuum environment is that when the electrical current starts, it ablates small amount of material from the wire's outer surface (either material from the wire bulk or, more likely, material adsorbed to the surface). Therefore, a low density plasma $\left(10^{14}-10^{16} / c c\right)$ is formed around the wire. This low density plasma has lower resistivity than the wire itself, so a substantial part of the current flows through the surrounding plasma rather than through the wire. The reduction in the current that flows through the wire prevents it from exploding (unless the current pulse is very intense). On the other hand, when the experiment is performed in atmospheric pressure the ablated material flows into the high density $\left(\sim 10^{19} / c c\right)$ air surrounding the wire. Hence, low density plasma cannot

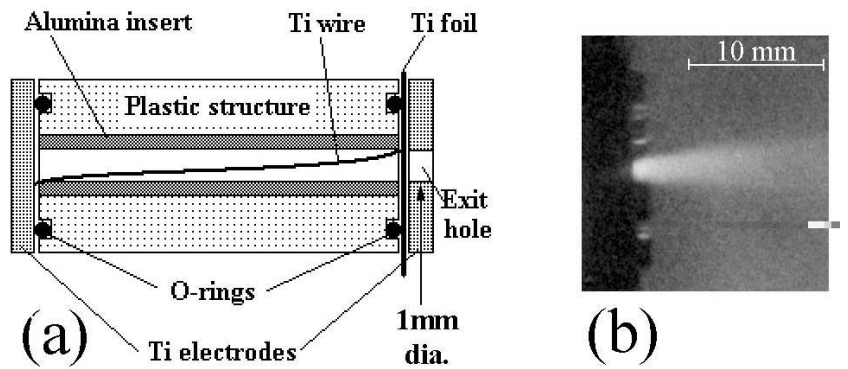

FIG. 2: (a) An illustration of the sealed capillary structure. The exit hole is sealed using a thin Ti foil that rips after the wire explosion. (b) Typical fast photography image of a plasma jet emerging from the sealed capillary device (in vacuum).

be formed and the current continues to flow through the wire leading to its explosion.

In order to enable the formation of a plasma jet in vacuum environment a change in the setup of the experiment was introduced. The inner volume of the capillary was sealed with atmospheric pressure inside, enabling the explosion of the wire. By properly selecting the inner diameter of the capillary and the diameter of the wire the plasma impurity caused by the atmospheric air was kept lower than $1 \%$. The seal of the capillary towards the exit hole was realized using a thin Ti foil $(12 \mu m)$. A schematic drawing of the sealed capillary is presented in figure 2 2 a. The foil is designed to stand a pressure difference of more than 1 atmosphere without ripping. However, After the wire explodes the high pressure formed in the capillary rips the foil, and a plasma jet flows out of the capillary. This capillary was inserted to vacuum environment (up to $10^{-6} \mathrm{Torr}$ ) and used to eject a jet of high purity $\mathrm{Ti}$ plasma. In all the experiments reported below we used a $1 \mathrm{~mm}$ inner diameter, $30 \mathrm{~mm}$ long Alumina capillary with a $125 \mu \mathrm{m}$ diameter Ti wire. The charging voltage of the $10 \mu F$ capacitor was $2 k V$. In all these experiments the electrical signals showed that the wire exploded properly, and a jet of Ti plasma emerged from the capillary. The properties of the Ti plasma jet emerging from the capillary were further investigated by means of fast photography and visible light spectroscopy.

Fast photography images with exposure time of 200ns were used to study the jet propagation. A typical fast photography image is displayed in figure $2 \mathrm{~b}$, showing the plasma jet formation outside the sealed capillary nozzle. The velocity of the plasma jet tip in vacuum was $4.5 \pm 0.5 \mathrm{~mm} / \mu \mathrm{s}$ (compared to $0.6 \pm 0.1 \mathrm{~mm} / \mu \mathrm{s}$ in similar experiments performed at atmospheric ambient pressure). Very directional jets with opening half-angle of $<15^{\circ}$ were produced. The plasma properties of the jet (electronic temperature and density) were measured using visible light spectrometery ${ }^{14}$. The fluorescence light from the jet was collected using an optical fiber with an appropriate collimator. The light was collected from jet at a distance of $1.5 \pm 1 \mathrm{~mm}$ from the exit hole. The spec- 
trum was time integrated during the first $80 \mu s$ after the plasma flow started (the long integration time was used in order to achieve good signal-to-noise ratio). Spectra taken at shorter integration durations showed similar results, since the flow of the plasma was steady at these times. We have performed measurements in several spectral regimes to observe different lines of $\mathrm{Ti}$ and its singly ionized ion. In all the measurements the majority of the lines were identified as Ti lines, which is an indication to the purity of the plasma. For a plasma in LTE the intensity ratio between two spectral line (of the same ion specie) is given by $\frac{15}{}$ :

$$
\frac{I_{1}}{I_{2}}=\frac{f_{u l}(1) g_{u}(1)}{f_{u l}(2) g_{u}(2)}\left(\frac{\lambda_{2}}{\lambda_{1}}\right)^{3} \exp \left[-\frac{E_{u}(1)-E_{u}(2)}{k_{B} T_{e}}\right]
$$

Where the indexes 1,2 stand for the two spectral lines, $I$ is the measured intensity, $f_{u l}$ is the absorption oscillator strength, $g_{u}$ is the statistical weight of the upper level, $\lambda$ is the wavelength of the transition, $E_{u}$ is the energy of the upper level of the transition and $k_{B}$ is the Boltzman constant. The plasma jet is at LTE since its electronic density is higher than the critical density (in our conditions $N_{c r} \cong 10^{14} / c c$ ). By comparing the intensities of the spectral lines $439.4 \mathrm{~nm}$ and $441.7 \mathrm{~nm}$ of natural $\mathrm{Ti}$, and using eq. 1 we calculated an electronic temperature of $1.5 \pm 0.5 \mathrm{eV}$.

The electronic density was determined by measuring the broadening of the $368.5 \mathrm{~nm}$ spectral line of singly ionized Ti. Several mechanisms contribute to the line-width of a spectral line, including natural broadening, Doppler broadening, Stark broadening as well as the spectrometer resolution. In the experimental setup we used, the dominant broadening mechanism for the $368.5 \mathrm{~nm}$ line was the Strak broadening (electron impact). Therefore the plasmas electronic density could be determined by measuring the FWHM of the $368.5 \mathrm{~nm}$ line. In figure 3 the measured spectrum in the vicinity of the $368.5 \mathrm{~nm}$ line is depicted.

The spectral line at $368.6 \mathrm{~nm}$ (from natural Ti) also appears in the spectrum, therefore a double peak curve (Voigt profile) was fitted to both lines (displayed in figure 3). From this measurement the electronic density of the plasma was found to be $N_{e}=(4 \pm 1.5) \times 10^{17} \mathrm{elec} / \mathrm{cc}$ (using electron impact parameter for the $368.5 \mathrm{~nm}$ line given in $\left.\frac{16}{6}\right)$. Similar results were achieved from the broadening of another spectral line. The mean ionization of $\mathrm{Ti}$ plasma at an electronic temperature of $1.5 \mathrm{eV}$ is $\bar{Z}=1.5$, therefore the density of $\mathrm{Ti}$ ions in the plasma jet is about
$N_{i}=(2.7 \pm 1) \times 10^{17}$ ion $/ c c$. The measured density at the exit hole of the capillary is an order of magnitude higher than the required density of the plasma target. By properly selecting the geometry of the secondary and main capillaries, a suitable plasma target can be achieved.

In conclusion, a simple technique to produce pure $\mathrm{Ti}$ plasma in vacuum environment was demonstrated. The technique is based on exploding a $125 \mu \mathrm{m}$ Ti wire inside a sealed capillary containing air. A thin Ti foil, which seals one of the capillary ends, rips after the wire explosion

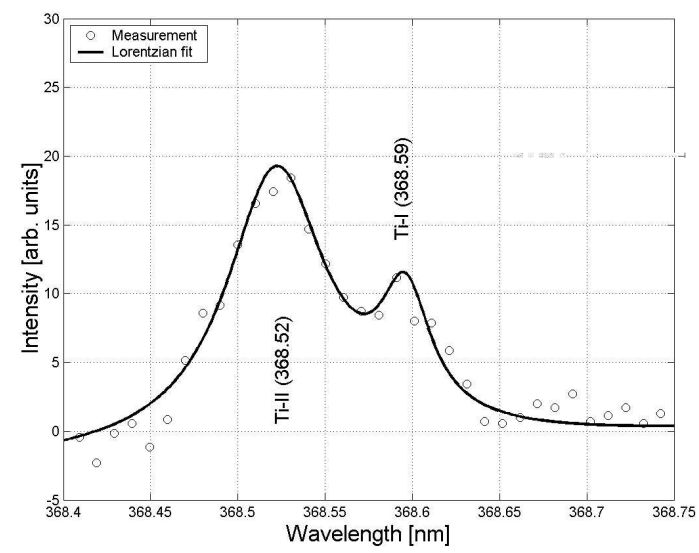

FIG. 3: Time integrated, space resolved spectrum of the Ti plasma jet emerging from the sealed capillary. The plasma electron density is inferred from Stark broadening of the TiII $368.52 \mathrm{~nm}$ spectral line.

and allows the plasma to flow out of the capillary. The Ti plasma jet formed in the experimental conditions used here had an axial velocity of $4.5 \pm 0.5 \mathrm{~mm} / \mu \mathrm{s}$, an opening half-angle of $<15^{\circ}$. A time integrated spectroscopy measurement performed $1.5 \pm 1 \mathrm{~mm}$ from the exit hole of the capillary showed that the plasma had an electron temperature of $T_{e}=1.5 \pm 0.5 \mathrm{eV}$ and an ion density of $N_{i}=(2.7 \pm 1) \times 10^{17} \mathrm{ion} / \mathrm{cc}$. By properly selecting the geometry, this plasma jet can be used to create a plasma target suitable for a Ne-like Ti capillary discharge XUV laser ${ }^{17}$. This technique for forming pure metallic plasma in vacuum might be useful for other applications such as a target for dense Z-pinch experiments.

This work was partially supported by the Fund for Encouragement of Research in the Technion. We acknowledge the helpful discussions with Ron Nemirovsky and the technical assistance of Yoav Erlich and Ofer Bokovza.
1 R. C. Elton, X-ray Lasers, (Academic Press, San-diego, 1990).

2 J. J. Rocca, V. N. Shlyaptsev, F. G. Tomasel, O. D. Cortazar, D. Hartshorn, J. L. A. Chilla, Phys. Rev. Lett., 73, 2192, (1994).

3 J. J. Rocca, Rev. Sci. Instrum., 70, 3799, (1999).
4 A. Ben-Kish, M.Shuker, R. Nemirovsky, A. Fisher, A. Ron, J. L. Schwob, Phys. Rev. Lett., 87, 015002-1, (2001).

${ }^{5}$ G. Niimi, Y. Hayashi, M. Nakajima, M. Watanabe, A. Okino, K. Horioka, E. Hotta, J. Phys. D: Appl. Phys., 34, 2123, (2001).

${ }^{6}$ G. Tomassetti, A. Ritucci, A. Reale, L. Palladino, L. Reale, 
S.V. Kukhlevsky, F. Flora, L. Mezi, J. Kaiser, A. Faenov, T. Pikuz, Eur. Phys. J. D, 19, 73, (2002).

7 M. Frati, M. Seminario, J. J. Rocca, Optics Letters, 25, 1022, (2000).

8 J. J. Rocca, O. D. Cortazar, F. G. Tomasel, B. T. Szapiro, Phy. Rev. E., 48, R2378, (1993).

${ }^{9}$ F. G. Tomasel, J. J. Rocca, V. N. Shlyaptsev, C. D. Macchietto, Phys. Rev. A, 55, 1437, (1997).

10 S. V. Kukhlevsky, L. Kozma, L. Palladino, A. Reale, Proc. SPIE 3156, 174, (1997).

11 A. Rahman, E. C. Hammarsten, S. Sakadzic, J. J. Rocca, J. F. Wyart, Physica Scripta, 67, 414, (2003).

12 R. Nemirovsky, A. Ben-Kish, M. Shuker, A.Ron, Phys. Rev. Lett., 82, 3436, (1999).
13 M. Shuker, A. Ben-Kish, R. Nemirovsky, A.Ron, Proceeding of the 6th Intl. Conference on X-Ray Lasers, Kyoto, 1998, edited by Y. Kato, H. Takuma and H. Daido, (IOP, Bristol, 1999) p. 187.

14 H. R. Griem, Plasma Spectroscopy, (McGrow-Hill, New York, 1964).

15 G. Bekefi, Principles of laser plasmas, (Wiley, New York , 1976).

16 V. H. Bartolic, Z. Andreic, H. J. Kunze, Physica Scripta, 50, (1994).

17 M. Shuker,M. Sc. Dissertation, Technion - Israel Institute of Technology, (2000). 